\title{
Naissance de la clinique allemande
}

Isabelle von Bueltzingsloewen

\section{SUMMARY}

The aim of this article is to show that, in the case of Germany, the birth of the clinic not only represents a change in the medical outlook, as described by Foucault, but should also be analyzed as the result of a complex interplay of political, economic, and social forces and as a conjunction of various demands which do not merely emanate from the university and scientific institutions.

\section{RÉSUMÉ}

Cet article vise à montrer dans le cas de l'Allemagne que la naissance de la clinique n'est pas seulement une mutation du regard médical comme l'a décrit Foucault mais doit être également analysée comme le produit du jeu complexe des forces politiques, économiques et sociales et comme la convergence de plusieurs demandes qui dépassent le seul cadre universitaire et scientifique.

Pour qui s'aventure, très prudemment, dans ce champ de recherche immense que constitue l'histoire de la médecine, la cohabitation avec Michel Foucault apparaît inévitable, à la fois pesante et stimulante. Rompant brutalement avec les méthodes et les conceptions traditionnelles, les travaux du philosophe ont ouvert des perspectives entièrement nouvelles; dans un contexte favorable marqué par la multiplication des travaux de démographie historique, par le développement de l'histoire des mentalités mais aussi par le rejet de l'autorité médicale qui caractérise les années 1970, ils ont sans aucun doute contribué à l'intérêt nouveau porté à la médecine, à la santé et à la maladie par des historiens français à la fois fascinés et irrités par les thèses foucaldiennes. Aujourd'hui encore, et bien que contesté, partiellement ou globalement, par de nombreux représentants de l'histoire sociale, l'apport et l'héritage de Foucault demeurent incontestables. 
La référence foucaldienne s'impose également, incontournable, dans le domaine plus restreint de l'histoire de la clinique; publiée voilà maintenant

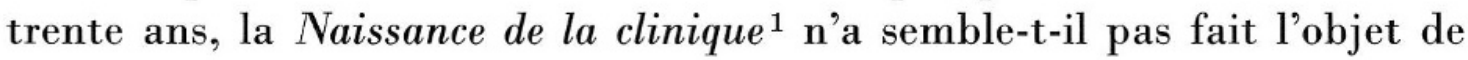
discussions et de polémiques aussi vives que celles suscitées par quelques autres publications majeures de l'auteur ${ }^{2}$. Sans doute le sujet, moins provocateur, s'y prêtait-il plus difficilement. Aussi l'approche alors très novatrice de Foucault dans ce domaine n'a-t-elle guère été renouvelée par des recherches récentes bien qu'elle ne soit pas sans poser un certain nombre de questions à l'historien.

L'histoire de la clinique n'a pas davantage mobilisé les chercheurs allemands. Mais ici l'explication est d'abord à chercher dans la tradition longtemps dominante d'une histoire de la médecine éminemment positiviste. Dans une démarche soucieuse de mettre en valeur les avancées glorieuses d'une médecine précisément définie par sa capacité à découvrir et à progresser, l'école clinique allemande du premier XIXe siècle faisait trop pâle figure au regard de l'école anatomo-clinique française qui, au faite de sa gloire, attire alors à Paris des médecins venus du monde entier. Quels trophées aligner qui puissent rivaliser avec le tableau de chasse de la clinique française? Quelles grandes figures opposer à la kyrielle des grands cliniciens français, à un Bichat, à un Pinel, à un Broussais, à un Laennec ou à un Corvisart? Il semblait décidément plus prudent de se tenir sur la réserve et d'insister plutôt sur les grandes réussites de la médecine de laboratoire qui, dans la seconde moitié du siècle, permirent aux médecins allemands de triompher à leur tour face à une recherche médicale française qui semble alors avoir perdu l'essentiel de son dynamisme ${ }^{3}$.

Sans doute ce «complexe d'infériorité» de la clinique allemande vis-à-vis de son homologue française n'a-t-il plus lieu d'être dans une conception plus sociale de l'histoire médicale telle qu'elle s'est imposée en Allemagne au début des années 1980 sous l'influence conjuguée des travaux des sociologues et historiens américains et français. Ce changement de perspective qui permit aux historiens allemands de se lancer sur le terrain fécond de la médicalisation de la société n'a pourtant pas renouvelé l'étude de la clinique. L'explication est sans doute à chercher du côté de l'approche foucaldienne elle-même qui rend difficile l'appréhension de la clinique allemande en tant qu'entité originale: pour Foucault il ne fait en effet aucun doute que la clinique est une «invention» française qui constitue une sorte de modèle unique et universel. Dès lors, toute clinique qui s'écarte de ce modèle absolu ne peut revendiquer le label de clinique véritable. La première difficulté 
consiste donc à qualifier cette réalité allemande qui, dès la fin du XVIIIe siècle et surtout pendant le premier XIXe siècle, se désigne elle aussi sous l'appellation de clinique mais qui pourtant n'a que très peu de choses en commun avec l'Ecole anatomo-clinique française. Sans doute Foucault la qualifierait-il de «proto-clinique», de «clinique première manière» ou encore de «clinique hippocratique», termes qu'il utilise par ailleurs pour désigner la clinique française du XVIIIe siècle dans un chapitre significativement intitulé «vieillesse de la clinique» ${ }^{4}$. Dans cette perspective, il semble donc que la clinique allemande ne puisse être abordée autrement qu'en terme de retard, n'ayant d'existence que négativement par rapport au modèle seul autorisé d'une clinique française hégémonique.

Notre propos ne vise aucunement à réhabiliter une clinique allemande injustement dépréciée ou à «déboulonner» une clinique française outrageusement encensée par l'historiographie mais au contraire à abandonner toute démarche «évaluative» qui se traduirait en terme d'avance et de retard, distribuant tour à tour bons et mauvais points. Tout d'abord en redonnant au mot clinique son sens le plus extensif, la clinique désignant de façon très large toute observation au lit du malade à laquelle est joint un enseignement; la clinique est donc toute à la fois un système pédagogique, une méthode d'analyse pathologique, un ensemble de données et de connaissances acquises par la mise en œuvre de cette méthode, enfin une institution qui peut être hospitalière mais aussi, comme dans le cas de l'Allemagne, non hospitalière. Le substantif de clinique n'étant guère employé, pas plus en allemand qu'en français, avant les années 1810-1830, il conviendrait d'ailleurs d'utiliser des termes plus précis tels que enseignement clinique, médecine clinique (ou médecine pratique) ou encore institut (ou hôpital) clinique ${ }^{5}$ qui permettent d'opérer une distinction plus nette entre les différentes acceptions du mot.

Trois autres correctifs sont également à apporter à la vision foucaldienne qui permettent de relativiser l'opposition tranchée qui semble exister, au moins jusqu'aux années 1840, entre une clinique française dynamique et novatrice et une clinique allemande routinière et rétrograde. Tout d'abord, la clinique française n'a pas tout inventé mais doit au contraire beaucoup aux emprunts étrangers qu'elle a su intégrer dans une perspective nouvelle ${ }^{6}$. D'autre part elle n'est pas une et indivisible mais abrite au contraire une multiplicité de courants qui laissent subsister des approches qui contredisent les principes localistes de la nouvelle médecine anatomo-clinique? Enfin, et c'est là sans doute l'aspect décisif, cette clinique française qui 
conserve encore bien des caractères anciens n'est pas apparue brutalement telle une révélation ou un miracle; sans aucun doute la rupture entre la médecine d'observation du XVIIIe siècle et la nouvelle médecine anatomoclinique a-t-elle été grandement exagérée, contribuant à renforcer le prestige, déjà inégalé, de la clinique française ${ }^{8}$.

Cette lecture critique de Foucault conduit à reposer en d'autres termes la question de la naissance de la clinique. En contestant d'abord l'emploi même du mot naissance qui implique un surgissement soudain pour préférer les mots de genèse ou d'émergence qui évoquent au contraire une séquence longue et heurtée. En quittant d'autre part le seul terrain scientifique et philosophique pour montrer que la genèse de la clinique doit certes être comprise comme une mutation du regard médical mais également et peutêtre surtout comme le produit du jeu complexe des forces politiques, économiques et sociales ${ }^{9}$.

Dans le cas de l'Allemagne, cette démarche, fondée sur l'étude du temps long, est en quelque sorte justifiée par l'absence du fait révolutionnaire; là encore l'opposition avec la France peut apparaître radicale, la clinique française étant habituellement présentée comme la fille d'une révolution qui permit de rompre le carcan de l'Ancien Régime médico-universitaire et de concrétiser rapidement des projets formulés dans la période précédente. En revanche, le développement de la clinique allemande apparaît comme le fruit d'une évolution lente, entamée dès la fin du XVIIe siècle puis poursuivie ensuite avec des phases d'accélération et de ralentissement mais sans rupture majeure, jusqu'à la fin des années 1820. Trois étapes, bien distinctes, peuvent être identifiées sans que l'on puisse fixer à chacune d'elles des limites très précises. Une première étape coïncide avec la fin du XVIIe siècle et la première moitié du XVIIIe siècle; pendant cette première période, la clinique demeure au stade d'idée et d'idéal sans parvenir à prendre corps. Dans une seconde étape qui concerne les années 1750 à 1790 environ, l'idée de clinique se concrétise dans quelques universités touchées par l'Aufklärung, Vienne, Fribourg, Göttingen ou encore Erlangen. Enfin dans une troisième étape qui s'étend des années 1790 à la fin des années 1820, le modèle clinique se répand dans l'ensemble des universités allemandes.

Cette chronologie une fois établie, il n'est pas possible d'isoler un seul élément déterminant dans la genèse de la clinique allemande; celle-ci s'explique au contraire par la convergence de facteurs multiples et interactifs. Le premier facteur à prendre en compte est la réforme de l'institution universi- 
taire. Si la clinique française s'est développée dans une structure institutionnelle nouvelle - les écoles puis les facultés de médecine qui remplacent les universités récemment abolies, l'émergence de la clinique allemande s'inscrit quant à elle dans un cadre permanent, l'université, qui a su s'ouvrir aux idées et aux pratiques nouvelles.

A la fin du XVIIe siècle, les universités allemandes ne semblent pourtant pas en état de stimuler et d'accueillir l'innovation, pas plus en médecine que dans les autres branches du savoir scientifique ${ }^{10}$. La plupart d'entre elles sont en perte de vitesse et font l'objet de critiques virulentes; on sait l'acharnement de Leibniz à condamner une institution dans laquelle il ne voit que le reliquat anachronique d'un passé obscur et à dénoncer pêle-mêle les méthodes de travail, le style des cours, le découpage des matières et en particulier la prééminence de la théologie qu'il juge inadaptés aux besoins nouveaux d'une société nouvelle. Pourtant de nombreux contemporains considèrent qu'il faut non pas abolir l'institution universitaire mais l'adapter aux besoins du temps en la soumettant à une réforme drastique; ce qui signifiait refondre sa structure et son organisation, redéfinir sa vocation, renouveler ses objets et ses méthodes. Seule une création ex nihilo pouvait supporter une telle rupture avec la tradition et c'est donc ainsi qu'il faut comprendre la fondation des universités de Halle en 1694 et de Göttingen en 1734/1737. Celle-ci se fait sur des bases et des principes entièrement nouveaux: l'université perd une grande partie de son autonomie et se trouve désormais placée sous la tutelle directe de l'Etat; la théologie perd sa prééminence sur les autres matières d'enseignement, laissant le champ libre au développement des autres facultés; enfin, l'université doit désormais prendre en charge la formation des serviteurs de l'Etat et ce y compris dans le domaine pratique en s'engageant à délivrer non pas seulement une Bildung mais une Ausbildung.

C'est dans ce contexte de régénération universitaire que se développent les premières tentatives d'organisation d'un enseignement de la médecine pratique. A l'université piétiste de Halle et sous l'influence directe de l'expérience menée par Hermann Boerhaave à Leyde, l'enseignement clinique est programmé dès 1708 et définitivement organisé en 1717 sous la houlette de Johann Juncker ${ }^{11}$. A Göttingen, le souci de compléter la formation des étudiants dans le domaine de la médecine pratique est clairement exprimé dans le projet élaboré en 1733 par Gottlieb von Werlhof, conseiller du curateur de l'université Gerlach Adolf von Münchhausen, qui servit de base à l'organisation de la nouvelle faculté de médecine ${ }^{12}$; celui-ci prévoit en 
effet de donner rang de discipline à la médecine pratique qui serait enseignée dans un hôpital dépendant de l'université.

Il fallut pourtant attendre les années 1750 pour qu'un embryon d'enseignement clinique se mette en place à Göttingen et le début des années 1780 pour que cet enseignement soit enraciné dans un cadre hospitalier. Jusqu'au milieu du XVIIIe siècle, l'expérience menée à Halle, dans le cadre très particulier du piétisme, resta donc isolée en Allemagne et ne permit pas l'établissement d'une véritable tradition clinique. Bien qu'indispensable à l'émergence et à la maturation d'idées et de méthodes nouvelles, la réforme de l'université n'avait donc pas suffi à sortir la clinique de l'âge expérimental; si le développement de l'enseignement clinique répondait à une demande formulée dans le milieu universitaire par quelques professeurs de médecine progressistes, cette demande ne trouvait guère d'échos dans la société environnante. C'est là sans doute, et même si d'autres éléments doivent être pris en considération ${ }^{13}$, ce qui explique principalement le décalage entre le projet développé par Werlhof, qui pourtant cadrait parfaitement avec les objectifs définis pour la nouvelle université, et sa réalisation.

Une telle contradiction met clairement en évidence l'insuffisance d'une approche exclusivement scientifique ou universitaire de la clinique. L'explication essentielle de l'échec à court terme du projet clinique est à chercher en dehors de l'université et plus précisément dans l'indifférence manifestée par le pouvoir et l'opinion publique vis-à-vis des questions de santé et de maladie, qui relèvent toujours de la sphère du privé voire de l'intime. Ce n'est que lorsque cette indifférence commence à céder que l'enseignement clinique, qui désormais répond à une demande non plus seulement scientifique mais socio-politique, s'implante plus solidement dans quelques universités ouvertes aux idées éclairées.

Ce nouvel intérêt pour les questions de santé correspond on le sait à une évolution de l'Aufklärung qui, abandonnant peu à peu l'orientation spéculative de ses débuts, devient un vaste mouvement de réformes qui touche la plupart des secteurs de la vie. L'un des signes les plus visibles de cette évolution est l'intervention plus grande des Etats dans le domaine de la médecine et de la prévention des maladies. Sans doute les Princes n'ont-ils pas attendu le milieu du XVIIIe siècle pour s'intéresser à la santé de leurs sujets; dès la première moitié du siècle, l'Etat se voit conférer, dans le cadre des nouvelles théories populationnistes, un rôle et une responsabilité de premier plan dans l'augmentation du nombre de ses sujets. Mais les projets 
et les déclarations d'intention l'emportent très largement sur les réalisations concrètes et il faut attendre les années 1750/1770 pour que l'action du pouvoir central se fasse à la fois plus déterminée et plus efficace.

Cette mobilisation, d'ailleurs limitée, de l'Etat en faveur de ce que l'on appelle alors la police médicale (medicinische Polizey) répond à la pression de plus en plus insistante des élites urbaines et en premier lieu à celle des médecins académiques; soucieux d'affirmer sa position socio-économique à un moment où son autorité n'est guère reconnue face à la concurrence de la médecine populaire et des guérisseurs mais aussi face à celle des sagesfemmes, des barbiers et des chirurgiens ${ }^{14}$, fortement dépendant d'une clientèle numériquement restreinte et issue de milieux généralement supérieurs au sien ${ }^{15}$, le médecin milite avec ardeur pour l'avènement d'une société médicalisée dans laquelle il occuperait une place de tout premier plan, en tant qu'éducateur du peuple et conseiller du Prince ${ }^{16}$. Dès lors, la Gesundheitsaufklärung peut se lire, du point de vue des médecins universitaires, comme une tentative de conquête d'un marché médical très disputé. S'il ne peut être encore question, dans un contexte de sous-encadrement médical chronique des populations, de revendiquer un véritable monopole, les médecins n'en manifestent pas moins leur désir d'affirmer leur emprise sur l'ensemble des populations et des personnels médicaux, grâce au concours de l'Etat et à la mise en œuvre d'une véritable campagne de sensibilisation à la santé à l'adresse du public bourgeois mais surtout des couches populaires ${ }^{17}$.

Reste que ce combat pour la reconnaissance de leur dignité, de leur autonomie et surtout de leur supériorité professionnelles passe nécessairement par ce que l'on appellerait aujourd'hui une obligation de résultat et les médecins académiques prennent alors cruellement conscience de leur impuissance thérapeutique: les «savoirs» du Medicus Purus ne peuvent en aucun cas rivaliser avec les «pouvoirs» des autres catégories de soignants. La caricature du jeune médecin publiée à titre anonyme par Friedrich Anton Mai en $1798^{18}$ est révélatrice de ce sentiment de désarroi éprouvé par les jeunes praticiens confrontés pour la première fois à la réalité de la maladie. D'où la remise en cause, quasi unanime, de la formation universitaire des médecins qui laisse le futur praticien totalement démuni face à ce qui constitue l'impératif premier de son métier: soigner ${ }^{19}$. Face aux lacunes d'un enseignement essentiellement livresque et théorique qui fait du médecin un Gelehrter, la revendication d'un savoir plus pratique se fait plus insistante: on réclame en particulier un enseignement plus systématique de l'anatomie 
mais surtout, reprenant les arguments déjà formulés au début du siècle, on préconise le développement d'un enseignement pratique au chevet du malade dont bénéficient déjà la plupart des chirurgiens formés dans des collèges médico-chirurgicaux ou dans des hôpitaux militaires. Dès 1746 , dans son Medicus Politicus, Friedrich Hoffmann insiste ainsi sur la nécessité d'instaurer un enseignement clinique dans toutes les universités ${ }^{20}$; dans son plan de réforme de l'enseignement médical dispensé par l'université de Vienne, présenté à l'impératice Marie-Thérèse en 1749, Gerhard van Swieten prévoit lui aussi une démonstration pratique au lit des malades; l'idée est également défendue par Albrecht von Haller et Samuel Tissot dans deux rapports adressés en 1765 au collège sanitaire de la ville de Berne. Quelques années plus tard, dans son Essai sur les moyens de perfectionner les études de médecine, Tissot demande plus généralement que la pratique de la médecine soit reconnue comme l'une des treize parties indispensables à l'étude de la médecine ${ }^{21}$. A la fin des années 1770 et plus encore à partir des années 1780 , la mobilisation en faveur de la rénovation des études médicales se fait plus massive gagnant l'ensemble de la classe médicale. De l'avis de tous, la reconnaissance sociale du médecin académique passe désormais par l'accroissement et le contrôle de ses connaissances et en particulier de ses compétences pratiques qui doivent faire de lui un expert de la maladie et de la médecine ainsi que le gardien de la santé publique.

Cette revendication de pouvoir et cette volonté d'améliorer la formation pratique des futurs médecins s'inscrit par ailleurs dans un contexte de «redécouverte» de la pauvreté qui mobilise l'ensemble des élites éclairées, aussi bien conseillers municipaux que pasteurs, curés, médecins et philanthropes en tout genre ${ }^{22}$. Dans la nouvelle logique - très optimiste - de l'Aufklärung, on dénie à la pauvreté tout caractère fataliste et on proclame la nécessité de la combattre et de la juguler. Or dans l'analyse des causes de la pauvreté, la maladie intervient en bonne place; c'est le moment où, sous l'influence de médecins réformateurs tels que Johann Peter Süssmilch, Leberecht Lentin ou Johann Peter Frank, on établit un lien direct entre pauvreté et morbidité. Dans cette optique, la maladie n’est plus seulement la conséquence d'un dérangement de l'équilibre des humeurs mais l'aboutissement inévitable de l'inégalité sociale qui plonge une partie de l'humanité dans la misère ${ }^{23}$. La lutte contre la maladie et la lutte contre la pauvreté apparaissent dès lors étroitement imbriquées; car en altérant les capacités physiques de l'individu, la maladie vient gravement perturber une logique de réintégration sociale qui repose sur le travail. 
Désignée comme l'un des objectifs prioritaires d'une politique d'assistance plus rationnelle et plus ambitieuse, la médecine des pauvres constitue pour les médecins académiques un nouveau champ d'investissement professionnel ${ }^{24}$ qui conforte l'argumentation des partisans d'une réforme de l'enseignement médical. A l'université, le jeune médecin devra être solidement initié à cette «nouvelle médecine» qui requiert des compétences spécifiques que le futur docteur devra nécessairement acquérir s'il veut, dans le cadre institutionnel des bureaux de bienfaisance, gagner la confiance des malades pauvres et combattre les «charlatans» sur leur propre terrain. Il devra ainsi apprendre à reconnaître et à soigner des pathologies spécifiques ${ }^{25}$ qu'il ne rencontre pas habituellement dans les couches aisées de la population et se familiariser avec un milieu social et des systèmes mentaux différents des siens, qu'il lui faudra apprendre à connaître pour mieux les combattre. En outre, si elle promet de nouveaux débouchés aux médecins académiques, l'institutionnalisation de la médecine des pauvres dans le cadre municipal a également fourni aux facultés de médecine le terrain nécessaire à la création d'instituts universitaires de médecine pratique. Le lien entre la rénovation de la politique d'assistance et le développement de l'enseignement clinique n'en apparaît que plus évident.

C'est donc dans ce climat de valorisation de la santé - individuelle et publique - et de réflexion sur la morbidité des plus pauvres que l'enseignement de la médecine pratique s'implante dans quelques universités. Celle de Vienne prend la tête du mouvement à partir des années $1750^{26}$; dans les années 1780 l'institution clinique est devenue le point de convergence d'une politique universitaire et d'une politique médico-sociale également ambitieuses. Ici, le projet clinique s'insère donc dans un programme de réformes global qui vise à soumettre l'ensemble de la société à l'emprise uniformisante de l'Etat. C'est l'Etat qui prend désormais en charge les pauvres et les malades, c'est lui qui assume également la formation pratique des futurs médecins. En 1768, la formule est étendue à l'université de Fribourg, avantposte de la domination autrichienne où, depuis 1751, les professeurs de la faculté de médecine réclamaient de se voir confier l'ensemble des malades soignés dans les hôpitaux de la ville afin d'initier leurs étudiants à la médecine pratique ${ }^{27}$. L'année suivante, un institut clinique universitaire s'ouvre également dans l'hôpital Julius de Würzburg.

A Göttingen, la chronologie du développement de l'enseignement clinique est plus difficile à établir et comporte plusieurs étapes. La première 
réalisation concerne en effet le seul secteur obstétrique: en 1751, le pouvoir central décide d'ouvrir une maison d'accouchement qui constitue aussitôt la vitrine de la faculté de médecine; mais cette clinique ne répondant guère aux besoins de formation pratique, plus généralistes, des étudiants en médecine, dès les années 1750 , quelques professeurs de médecine, tablant sur la demande médicale d'une population pauvre dont la municipalité ne s'était encore guère préoccupée, décident de créer des «collèges cliniques» privés ${ }^{28}$; ainsi en 1754 Rudolf A. Vogel prend-il l'initiative de convoquer à son domicile, deux fois par semaine, des pauvres malades afin de les examiner en présence de ses étudiants; il organise également de petites excursions en ville et dans la campagne environnante afin de visiter les malades incapables de se déplacer. La formule, encore très précaire, est reprise par Johann G. Roederer à la fin des années 1750 puis par Philipp G. Schroeder de 1767 à 1772. Mais ce n'est qu'en 1773, soit vingt ans après la première expérience tentée par Vogel, que Ernst G.Baldinger obtient la reconnaissance officielle de son clinicum ainsi qu'une subvention étatique de 200 thaler par an. A cette date, l'enseignement clinique est véritablement et définitivement intégré à l'institution universitaire.

L'organisation d'un enseignement clinique de type hospitalier constitue une étape supplémentaire. Jusqu'ici en effet, la ville ne possédant aucun hôpital susceptible d'accueillir un institut clinique, l'enseignement de la médecine pratique s'apparentait tout à fait à ce que l'on appelle aujourd'hui la médecine de ville. Il fallut attendre 1781 et le soutien financier de la loge franc-maçonne Augusta zu den drei Flammen, qui rassemblait une partie des élites éclairées de la ville gagnées à un projet qui présentait une double vocation sociale et scientifique, pour que soit créé un hôpital directement dépendant de l'université et spécialement destiné à répondre aux besoins de l'enseignement clinique ${ }^{29}$.

En dépit d'un fondement commun, le projet clinique revêtit donc des formes institutionnelles diverses selon les conditions locales. Des facteurs tels que la taille de la ville, la présence ou au contraire l'absence d'établissements hospitaliers préexistants, la structure et la vocation de ces hôpitaux ${ }^{30}$, l'état des rapports entre l'université et la municipalité, le pouvoir d'intervention des autorités centrales ou encore la personnalité des professeurs de clinique eurent souvent davantage d'influence sur les choix institutionnels opérés par les universités que les débats théoriques ${ }^{31}$. Au total et alors que la clinique française trouve sa traduction institutionnelle dans une structure unique, la clinique hospitalière, son «homologue» germanique a donc 
inventé des formes beaucoup plus variées; à la clinique hospitalière, installée dans un hôpital municipal préexistant s'ajoute en effet l'hôpital académique ${ }^{32}$ financé et géré par l'université et dont la vocation pédagogique est clairement désignée et surtout la policlinique, clinique non hospitalière qui s'apparente directement aux instituts de médecine à domicile (Krankenbesuchanstalten) créés depuis les années 1780 dans de nombreuses villes du nord de l'Allemagne sur le modèle de Hambourg ${ }^{33}$.

Reste que quelque fut la formule adoptée, l'enseignement clinique connut aussitôt un succès massif auprès des étudiants qui conduisit un nombre grandissant d'universités à emprunter cette voie ${ }^{34}$. Ainsi, dès le tournant du XVIIIe siècle, la nécessité d'un enseignement clinique ancré dans les universités était-elle un fait acquis et la tradition clinique solidement établie; mieux, l'enseignement clinique était clairement désigné comme le fer de lance de l'enseignement médical dans un climat de compétition universitaire accru et de plus grande mobilité des professeurs et des étudiants. En 1803, tout juste nommé professeur de médecine pratique à l'université de Göttingen, Karl Gustav Himly pouvait ainsi déclarer: «La nécessité de créer des instituts cliniques pour la formation des futurs médecins et chirurgiens est à ce jour tellement évidente et à ce point reconnue par tous qu'il n'est plus utile d'en apporter la preuve.» ${ }^{35}$ La grande réforme universitaire engagée dans la plupart des Etats allemands pendant ou après la période d'occupation napoléonienne confirma cette évolution et, donnant un nouveau souffle à l'enseignement clinique, permit son extension à l'ensemble des facultés de médecine. Là encore, la modernisation de l'institution universitaire, dans un contexte de forte augmentation du nombre des étudiants en médecine et d'allongement de la formation médicale, apparaît donc comme la condition, non suffisante certes, mais néanmoins nécessaire du développement de l'enseignement clinique. Les universités nouvellement créées, celle de Berlin en 1810, de Bonn en 1819 ou encore de Munich en 1826 disposèrent d'emblée d'instituts cliniques, à la fois de type hospitalier et policlinique. Dans quelques petites universités faiblement dotées ou réformées tardivement telles que celle de Giessen, de Greifswald ou de Rostock, l'évolution se fit il est vrai plus lentement ${ }^{36}$. Mais à la fin des années 1820 , le réseau allemand des cliniques universitaires était achevé; à cette date, tout étudiant en médecine allemand avait la possibilité - et dans certains cas l'obligation - de suivre un enseignement clinique avant d'entamer sa carrière de praticien. 
La naissance de la clinique allemande - c'est-à-dire à la fois d'une nouvelle institution à vocation pédagogique et scientifique et d'un nouveau regard sur le corps et la maladie - doit donc être comprise comme la convergence de plusieurs facteurs et de plusieurs demandes qui, de plus en plus pressantes, finirent par se rejoindre et se confondre: d'une part et dans l'ordre chronologique, une demande universitaire, de nature scientifique et pédagogique, suscitée par l'évolution des exigences en matière de formation médicale qui s'exprime dès le début du XVIIIe siècle. Cette demande, interne à l'université, ne trouve guère d'échos avant les années 1750-1780; à cette date, elle rencontre une demande plus large, de nature populationniste, exprimée par l'Etat et les ténors de la Gesundheitsaufklärung qui réclament de nouveaux experts chargés de veiller sur la santé privée et publique; cette demande est stimulée et relayée par les médecins académiques et par les étudiants en médecine - qui, sollicités par des universités rivales, jouèrent sans aucun doute un rôle considérable dans le développement de l'enseignement clinique - conscients des avantages qu'ils pourraient retirer de l'élargissement de leur sphère d'intervention en même temps que des insuffisances de leur formation pratique. Enfin, à partir des années 1780, une demande municipale se fait jour qui émane de l'ensemble des élites urbaines soucieuses de redéfinir la politique socio-urbaine et de réorganiser des bureaux de bienfaisance dans lesquels prendrait place une nouvelle catégorie de médecins chargés de préserver et de restaurer la santé des membres les plus pauvres de la communauté.

Sans doute ces évolutions ne sont-elles pas spécifiques à l'Allemagne. Pourtant, jusque dans les années 1840, même si elle ne demeura pas fermée aux évolutions, comme en témoignent par exemple le développement de la nouvelle école de Schönlein à partir des années 1820 ou de la seconde école viennoise, la clinique allemande ne s'orienta que très lentement vers une conception localiste de la maladie, fondée sur le développement systématique de l'anatomo-pathologie et de l'autopsie. L'explication n'est pas à chercher du côté d'une méconnaissance des nouvelles méthodes de la clinique française - les jeunes médecins allemands furent nombreux à fréquenter les hôpitaux parisiens ${ }^{37}$ - mais bien plutôt de la difficulté à intégrer des idées et des pratiques nouvelles dans un système cohérent de représentation du corps et de la maladie détaché de toute contingence organique, l'exemple le plus significatif de cette difficulté étant sans aucun doute la très lente diffusion du stéthoscope dans le monde médical allemand, y compris dans les 
instituts cliniques. Incontestablement il existe alors, malgré une grande diversité interne - pas plus que la clinique française la clinique allemande ne forme un tout homogène -, un modèle national allemand de clinique en grande partie imperméable aux influences françaises et fondé sur un certain nombre de traits spécifiques à l'espace germanique. Tout d'abord l'extrême dispersion des centres universitaires qui contraste radicalement avec la centralisation de la nouvelle clinique française. Souvent installées dans de petites villes, les facultés de médecine ne disposaient pour la plupart que d'un parc hospitalier restreint, mal adapté à l'observation sérielle et à la multiplication des autopsies. D'autre part, les hôpitaux dépendaient dans la majorité des cas des seules municipalités et aucun souverain ne fut en mesure, tel Napoléon, de les «offrir» aux facultés de médecine et aux cliniciens; les relations entre universités et municipalités furent dans la plupart des cas difficiles, butant sans cesse sur des conflits de pouvoir et de compétence qui freinèrent le développement d'un véritable secteur clinique. Enfin, et c'est là également un élément décisif, les facultés de médecine allemandes sont toujours demeurées dans le sein de l'université et n'existèrent par conséquent que dans leur rapport, complexe et changeant, aux autres facultés et à une idéologie dominante qui détermina, en partie tout au moins, la place modeste de l'enseignement clinique dans les études médicales allemandes ${ }^{38}$. La réforme néo-humaniste a sans aucun doute renforcé la dérive théorisante et spéculative de la médecine d'alors et a conforté la rupture entre théorie et pratique à l'intérieur du cursus médical. Dans ce contexte et dans la continuité de la période utilitariste ${ }^{39}$, la clinique conserva donc une vocation essentiellement pédagogique au détriment de sa dimension scientifique; l'enseignement clinique était en effet toujours considéré par une majorité de professeurs de médecine comme un simple champ d'application ou de vérification pratiques de connaissances énonçables théoriquement et non pas comme la source d'un savoir particulier.

Au total, l'histoire de la clinique est en tout cas indissociable d'une histoire plus générale, celle de la médicalisation de la société. Une médicalisation venue d'en haut mais également d'en bas. Le développement de l'enseignement clinique semble en effet révéler l'existence d'un marché médical en pleine mutation qui nourrit les espoirs de promotion des médecins académiques. En réclamant de l'Etat l'organisation d'une formation mieux adaptée aux impératifs pratiques de leur fonction, les médecins académiques et les étudiants en médecine auraient ainsi répondu à une pression venue du bas et réagi à l'apparition d'une demande accrue en 
prestations médicales bien avant le développement de l'industrialisation, de l'urbanisation et du paupérisme; c'est ce qui expliquerait également le développement des médecines parallèles mais aussi le succès massif rencontré par les premiers instituts cliniques auprès des malades, attirés par une politique de soins gratuits et plus généralement par les nouvelles institutions de soins mises en place par les municipalités dont le recrutement, contrairement à une idée trop répandue, dépasse largement le seul cercle des marginaux et des miséreux pour embrasser l'ensemble des couches populaires. Une théorie qui là encore vient contrarier les thèses de Michel Foucault et celles de la plupart des sociologues américains des années 1970 qui décrivent généralement la médicalisation comme un processus répressif imposé à une population très largement hostile et dont les aspirations n'auraient aucunement été prises en compte. Et qui surtout place au centre du processus un acteur de tout premier plan qui probablement a été le véritable arbitre de la situation: le malade. 


\section{Notes et références}

1 M. Foucault, Naissance de la clinique: une archéologie du regard médical, Paris, 1963. L'ouvrage a connu cinq rééditions entre 1963 et 1990.

2 En particulier Folie et déraison: histoire de la folie à l'âge classique paru en 1961 et surtout Surveiller et punir publié en 1975.

3 C'est ce qui ressort en tout cas de l'étude des très nombreux manuels allemands d'histoire de la médecine destinés aux étudiants en médecine.

4 Chapitre IV.

5 Dans l'Allemagne du XVIIIe siècle, on trouve également le terme de Clinicum qui désigne non pas la clinique dans son sens global mais l'institution clinique.

6 Voir 0 . Keel, «La place et la fonction des modèles étrangers dans la constitution de la problématique hospitalière de l'Ecole de Paris», History and Philosophy of the Life Sciences, 6, 1, 1984, pp. 41-73.

7 Ainsi le vitalisme, le néo-hippocratisme et la médecine psycho-sociale parviennent-ils à survivre pendant tout le siècle; voir J. Leonard, «La pensée médicale au XIXe siècle», in J. Leonard, Médecins, malades et société, Paris, 1992, pp. 217-240.

8 Voir: M. J. Imbault-Huart, «Les chirurgiens et l'esprit chirurgical en France au XVIIIe siècle», Clio Medica, 15, 3/4, 1981, pp.143-157.

9 C'est ce que nous avons tenté de faire tout au long de notre thèse de doctorat intitulée Enseignement clinique et médicalisation de la société dans l'Allemagne des XVIIIe et XIXe siècles soutenue à Lyon en décembre 1992.

10 Sur la situation des universités allemandes au tournant du XVIIe et au XVIIIe siècles voir: C. Mc Clelland, State, society, and university in Germany, 1700-1914, Cambridge, 1980, et N. Hammerstein, «Die deutschen Universitäten im Zeitalter der Aufklärung», Zeitschrift für Historische Forschung, 1983, pp. 73-89.

11 Sur les origines de l'enseignement clinique à l'université de Halle voir: H. H. Eulner, «Die Einrichtungen der Medizinischen Fakultät und ihre Geschichte», 450 Jahre Martin-Luther Universität Halle-Wittenberg, tome 2, Halle, 1952, pp.485-492, W. Kaiser et alii, «Collegium Clinicum Hallense», 250 Jahre Collegium Clinicum Hallense 1717-1967, Halle, 1967, pp. 9 76, enfin, «275 Jahre Versorgungs- und Lehrkrankenhaus Halle», Historia Hospitalium, 15, 1983-1984, pp. 243-272.

12 P.G. von Werlhof, «Gutachten wegen einer medicinischen Fakultät», 16 décembre 1733, in E. Roessler, Die Gründung der Universität Göttingen, Göttingen, 1855 (réédité à Darmstadt en 1987), pp. 298 et suivantes.

13 Les arguments financiers en particulier ne doivent pas être négligés; les crédits dont dispose la nouvelle université ne permettent en effet que de réaliser les projets considérés comme prioritaires; or on sait que c'est la faculté de droit qui constitue la colonne vertébrale de l'université alors que la faculté de médecine, qui n'attire qu'une minorité d'étudiants nobles, est longtemps demeurée au rang de parent pauvre; par ailleurs, à l'intérieur de la faculté de médecine, c'est l'anatomie qui est considérée comme la discipline prépondérante et l'essentiel des crédits va donc à la construction d'un théâtre anatomique inauguré dès 1738 . 
14 Sur le malaise de la médecine académique à la fin du XVIIIe siècle voir: U. Frevert, Krankheit als politisches Problem, 1770-1880, Göttingen, 1984, et C. Huerkamp, Der Aufstieg der Ärzte im 19. Jahrhundert, Göttingen, 1985.

15 Sur la relation de patronage qui unit les médecins à leurs malades voir: C. Huerkamp, «Ärzte und Patienten. Zum strukturellen Wandel der Arzt-Patient Beziehung vom ausgehenden 18. bis zum frühen 20. Jahrhundert», in A. Labisch et R. Spree (dir), Medizinische Deutungsmacht im sozialen Wandel des 19. und 20. Jahrhunderts, Bonn, 1989 pp. 57-74.

16 C'est ce qui ressort en particulier de l'étude des nombreuses topographies médicales publiées pendant la période. Voir J. Brügelmann, Der Blick des Arztes auf die Krankheit im Alltag (1779-1850), Berlin, 1982. Cette «utopie totalitaire» est également très présente chez les médecins éclairés français membres de la Société Royale de Médecine.

17 C'est dans ce cadre que s'inscrit l'Avis au peuple sur sa santé publié en 1761 par Samuel Tissot ainsi que les multiples almanachs et les catéchismes de santé étudiés par I.Sahmland, «Der Gesundheitskatechismus - ein spezifisches Konzept medizinischer Volksaufklärung», Sudhoffs Archiv, 75, 1991, pp.58-73.

18 (F. A. Mai), Stolpertus, ein junger Arzt am Krankenbette, Mannheim, 1798.

19 Voir en particulier le réquisitoire que dresse Johann Peter Frank dans le tome 1, p. 70 de son System einer medicinischen Polizey publié en 1779.

20 F. Hoffmann, Medicus politicus, Halle, 1746, tome 1, p. 6.

21 S. Tissot, Essai sur les moyens de perfectionner les études de médecine, Lausanne, 1785, p. 17.

22 Voir R. Endres, «Das Armenproblem im Zeitalter des Absolutismus», in F. Kopitzsch (dir), Aufklärung, Absolutismus und Bürgertum in Deutschland, Munich, 1976, pp.220-241, et U.Hermann (dir), "Das pädagogische Jahrhundert», Volksaufklärung und Erziehung zur Armut im 18. Jahrhundert in Deutschland, Weinheim, 1981.

23 Telle est la démonstration que fait Johann Peter Frank dans sa Akademische Rede vom Volkselend als der Mutter der Krankheiten publiée à Pavie en 1790. La plupart des autres médecins réformateurs ne mettent pas aussi radicalement en cause les fondements inégalitaires de la société.

24. Voir surtout J.A. Garn, Unmassgebliche Vorschläge zur Errichtung einer öffentlichen Krankenpflege für Arme jeden Orts und zur Abstellung der Kuren durch Afterärzte, Wittenberg, 1789, et C.E. Fischer, Versuch einer Einleitung zur medicinischen Armenpraxis, Göttingen, 1799.

25 C'est ce qui explique à cette période la floraison des manuels de pharmacopée destinés au traitement des maladies des pauvres.

26 Sur la mise en place de l'enseignement clinique à Vienne voir: G. B. Risse, «Clinical Instruction in Hospitals: The Boerhaavian Tradition in Leyden, Edinburgh, Vienna and Pavia», Clio Medica, 21, 1987-1988, pp.1-20, E. Lesky, «The Development of Bedside Teaching at the Vienna Medical School from Scolastics Times to the Special Clinics», in C.D. O'Malley (dir), The History of Medical Education, Berkeley, 1970, pp.217-234, et C.Probst, Der Weg des ärztlichen Erkennens am Krankenbett. Hermann Boerhaave und die ältere Wiener medizinische Schule (1701-1787), Wiesbaden, 1972.

27 Sur la création de l'Institut clinique de Fribourg voir: L. Heilmacher, Tradition und Zielsetzung der medizinischen Universitätsklinik in Freiburg, Stuttgart, 1951, et H.U. Kuermann, Das clinische Hospital der Stadt Freiburg/Breisgau, Fribourg, 1980. 
28 Voir: R. Kumsteller, Die Anfänge der medizinischen Poliklinik zu Göttingen, Göttingen, 1958.

29 Voir: H. Winkelmann, Das academische Hospital in Göttingen von 1781 bis 1850, Göttingen, 1981, thèse de médecine dactylographiée.

30 Les hôpitaux les plus anciens, souvent très petits et dont la fonction est plus sociale que thérapeutique conviennent en effet moins bien à l'enseignement clinique que les hôpitaux nouvellement créés ou rénovés. Dans certains cas, l'université a donc pu être amenée à créer un hôpital propre malgré l'existence dans la ville d'un autre établissement hospitalier. C'est en particulier le cas à Tübingen, à Giessen ou à Königsberg. Voir: I. v. Bueltzingsloewen, Enseignement clinique et médicalisation de la société dans l'Allemagne des XVIIIe et XIXe siècles, op. cit., pp. 273-302.

31 La question débattue étant de savoir quel type de clinique convenait le mieux à l'instruction des futurs praticiens.

32 Ou hôpital clinique, en quelque sorte l'ancêtre de nos CHU français.

33 Ces Krankenbesuchanstalten firent on le sait l'objet d'un débat passionné dans lequel s'opposaient partisans et adversaires de l'hôpital.

34 Il n'est pas question ici de restituer avec précision la chronologie de création des premiers instituts cliniques dans l'ensemble des universités allemandes. Cette démarche demanderait d'ailleurs une enquête minutieuse, les études menées jusqu'ici ayant négligé la complexité et la variété du phénomène. Ainsi ne prennent-elles généralement en compte que les instituts cliniques officiellement reconnus et subventionnés alors que ceux-ci ont très souvent été précédés par des instituts privés, souvent éphémères et qui par conséquent laissent peu de traces documentées. D'autre part ces travaux négligent généralement les instituts de type policlinique pour privilégier les instituts de type hospitalier.

35 K.G. Himly, Verfassung der öffentlichen medicinisch-chirurgischen Anstalt zu Göttingen, Göttingen, 1803, p.5.

36 Giessen ne dispose d'une clinique hospitalière qu'à partir de 1824; celle-ci est, jusqu'en 1831, financée par des crédits privés. A Greifswald il faut attendre 1825 et à Rostock 1828.

37 Voir U.Geigenmüller, Aussagen über die französische Medizin der Jahre 1820-1847 in Reiseberichte deutscher Ärzte, Berlin, 1985.

38 Contrairement à son collègue français qui, dès le début de son cursus, est introduit dans les hôpitaux pour suivre des leçons de clinique, l'étudiant allemand n'est mis que tardivement en contact avec les malades et la maladie.

39 Et dans la tradition de Boerhaave à laquelle les cliniciens allemands sont demeurés très longtemps fidèles.

Dr. I. von Bueltzingsloewen

MRASH - Centre Pierre Léon

12 , avenue Berthelot

F-69007 Lyon 